\title{
Development of fuzzy fractal representation of the image
}

\author{
D G Privezentsev ${ }^{1}$, A L Zhiznyakov ${ }^{1}$ and E V Pugin ${ }^{1}$ \\ ${ }^{1}$ Murom Institute, Vladimir State University, Orlovskaya str. 23, Russia, 602264
}

\begin{abstract}
The article considered the way of the fuzzy image representation with using fractal models of images. According to it, the image presented in the form of a tree range blocks which correspond to image blocks. Everyone range block represents the structure describing similarity of this block to another block of the image. As a result, it turns out treelike fractal representation of the image describing the distribution of property of self-similarity in the image. The basic possibilities of construction of algorithms of digital processing of the images which based on given representation are listed
\end{abstract}

\section{Introduction}

Most methods of digital image processing (including defectoscopy images) refer to one of two groups: spatial or frequency. Spatial methods make it possible to identify an object in an image uniquely, and frequency methods allow one to adequately analyse the spectral components of a two-dimensional signal (useful component and noise). In this case, spatial methods, as a rule, are subject to interference and require the source signal to be cleaned of noise, possibly with the loss of useful information. For frequency methods, the main problem is the localisation of objects of interest in the coordinate system of the image.

The use of space-frequency approaches, for example, based on wavelet transforms, allows you to detect objects in images against background noise more accurately. This approach is since the wavelet spectrum contains complete information about the original signal, including the spatial coordinates of the frequency components, and at the same time separates the useful and noise components.

However, the overwhelming majority of digital image processing algorithms based on the algorithms for extracting useful information, which often does not allow the technical vision systems to solve non-trivial problems. In this regard, it is proposed to combine the mathematical theory of fuzzy sets and fuzzy logic with the proven fractal methods of digital image processing.

To develop a system of new features, need to create a new model of a digital image. It is proposed to modify the fractal model developed by the project manager by using a fuzzy distance in it as a measure of similarity of the image areas. This modification allows expanding the hierarchy of representations of the original image, thereby increasing the amount of useful information about the original image.

With increasing complexity of the tasks solved, it is expedient to use the specially oriented methods that to the construction of models, methods and algorithms that take into account the incompleteness and inaccuracy of the initial data. It is in such situations that the theory of fuzzy sets and fuzzy logic proves to be the most constructive. Already achieved theoretical results allow 
to consider fuzzy logic as a promising tool for the analysis of heterogeneous information, in the form of complex qualitative linguistic descriptions and quantitative data. In modern works, we considered methods and algorithms for using fuzzy logic and fuzzy set theory to solve problems of digital image processing.

Analysis of the used fuzzy image processing schemes $[1,2,3]$ shows that the used fuzzy representations of images, as well as the membership functions used to translate images into fuzzy forms, do not have a single mathematical basis. Used fuzzy signs and properties of images built on the representation of images in the form of sets. In this case, there is no possibility of sharing fuzzy technologies with classical algorithms of digital image processing. Some papers describe fuzzy measures, fuzzy integrals used in segmentation and clustering problems as local statistical features defined on the set to which the image[4]. From this, it follows that for the joint use of classical features, measures and algorithms in conjunction with fuzzy imaging technologies, a system of fuzzy signs, measures and distances needed that allows developing new algorithms for digital image processing.

In connection with the preceding, it is proposed to develop a fuzzy mathematical model of a digital image based on its description by the terms of fuzzy sets using fuzzy logic, which is the basis for constructing new algorithms for extracting information about features and image processing algorithms.

\section{Fractal Model of Digital Image}

The fractal model of the digital image developed and described in $[5,6,7]$ implements the system of iterated functions and can be represented by the expression

$$
f^{\prime}=\sum_{k=1}^{n}\left(B_{n_{i}, m_{i}}^{r_{i}}\right)^{*}\left[s_{i}\left(\tilde{w}\left(B_{k_{i}, l_{i}}^{d_{i}}\right)[f]\right)+o_{i}\right] .
$$

where

$$
D=\left(B_{k_{i}, l_{i}}^{d_{i}}\right)[f]
$$

is an operator of extracting a domain block with the index $d_{i}$ from the original image $f$. The transformation that performs the change in the color characteristics of the domain block and the geometric transformation has the following form

$$
D^{\prime}=s_{i}(w[D])+o_{i} .
$$

After all the transformations are performed on the original domain block, the result is inserted into the image

$$
f^{\prime}=\sum_{i=1}^{M}\left(B_{n_{i}, m_{i}}^{r_{i}}\right)^{*}\left[D^{\prime}\right]
$$

Using 1, 2 and 3, formulate an expression describing the range block of the image through the domain block

$$
R_{i}=D^{\prime}=s_{i}(w[D])+o_{i} .
$$

For comparison of two images $f, h$ metric used RMS:

$$
g(f, h)=\sum_{i, j} \sqrt{\frac{1}{m \cdot n}[f(i, j)-h(i . j)]^{2}},
$$

where $m, n$ - images size. For each range block of the image $R_{i}$, the domain block $D_{i}$ is selected which best approximates the range block. In other words, there is a pair $\left(R_{i}, D_{j}\right)$ for which the metric (6) is minimal. 
To generate a fractal image code, we used a quadtree of range blocks. The root of the tree is the image itself.

Expression 5 can be represented as follows

$$
\begin{gathered}
R_{i}=D^{\prime}+\xi_{i}, \\
\xi_{i}=g\left(R_{i}, D^{\prime}\right) .
\end{gathered}
$$

In other words, the fractal model describes the original image using the quadtree. Each leaf of a tree is a range block of the image, which corresponds to a domain block so that the approximation error is minimal:

\section{Intuitionistic Fuzzy Set}

A fuzzy set $A$ in a finite set $X=x_{1}, x_{2},, x_{n}$ may be represented mathematically as

$$
A=\left\{\left(x, \mu_{A}(x)\right) \mid x \in X\right\}
$$

where the function $\mu_{A}(x): X \rightarrow[0,1]$ is the measure of the degree of belongingness or the membership function of an element $x$ in the finite set $X$.

An Intuitionistic Fuzzy Set $A$ in a finite set $X$ may be mathematically represented as

$$
A=\left\{\left(x, \mu_{A}(x), \nu_{a}(x)\right) \mid x \in X\right\}
$$

where the functions $\mu_{A}(x), \nu_{A}(x): X \rightarrow[0,1]$ are, respectively, the membership function and the non-membership function of an element $x$ in a finite set $X$ with the necessary condition

$$
0 \leqslant \mu_{A}(x)+\nu_{A}(x) \leqslant 1 .
$$

It is clear that every fuzzy set is a particular case of IFS:

$$
A=\left\{\left(x, \mu_{A}(x), 1-\mu_{A}(x)\right) \mid x \in X\right\} .
$$

\section{Development of fuzzy fractal model}

To use all the features of fuzzy sets is necessary to modify the model (5) and bring it to the fuzzy mind. During the construction of the fractal image code, a set of range blocks, domain blocks and approximation error values are generated:

$$
R=\left\{R_{i}\right\}, R_{i}=\left\{D_{j}, \xi_{i, j}\right\} .
$$

Then, using 8, expression 9 can be expressed using a fuzzy set

$$
R=\left\{D_{j}, \mu_{R}\left(D_{j}\right), 1-\mu_{R}\left(D_{j}\right) \mid D_{j} \in D\right\} .
$$

Then the membership function $\mu_{R}\left(D_{j}\right)$ may be represented as

$$
\mu_{R}\left(D_{j}\right)=\xi_{j} / \xi_{\max },
$$

where $\xi_{\max }$ the maximum approximation error, which is defined as the difference between absolutely black and absolutely white images:

$$
\xi_{\max }=255 / \sqrt{m \cdot n},
$$

where $m, n$ - image size. 
Let the image $\mathrm{f}$ is a graph $G=(V, \bar{U})$, where $V$ is the list of range blocks of the image, $\bar{U}$ is the connections between the range blocks. Then the process of forming a tree of range blocks of the image can be described as follows. There is a range block of zero level $R^{0}$, which corresponds to the whole image and $v_{1}=R^{0}$. It contains range blocks of the first level $R_{1}^{1}, R_{2}^{1}, R_{3}^{1}, R_{4}^{1} \in R^{0}$ :

$$
\begin{gathered}
v_{2}=R_{1}^{1}, v_{3}=R_{2}^{1}, v_{4}=R_{3}^{1}, v_{5}=R_{4}^{1}, \\
\overline{u_{1}}=\left(v_{1}, v_{2}\right), \overline{u_{2}}=\left(v_{1}, v_{3}\right), \overline{u_{3}}=\left(v_{1}, v_{4}\right), \overline{u_{4}}=\left(v_{1}, v_{5}\right) \in \bar{U} .
\end{gathered}
$$

Continuing the formation of the fractal code, a complete tree of range blocks is obtaining, from which the image is constructed. A fragment of the tree of range blocks is shown in Fig. 1.

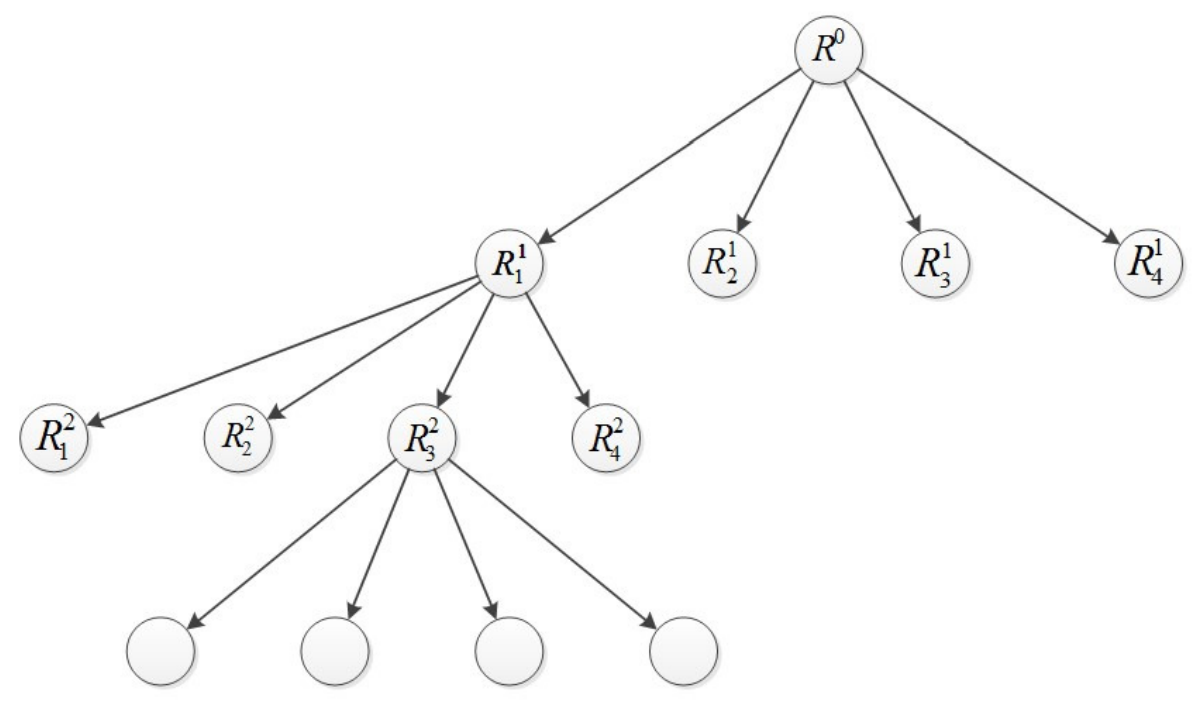

Figure 1. Fragment of the tree of range blocks.

The operator for the formation of a fractal code called a direct fractal transformation:

$$
F(f, D)=\Phi
$$

where $\Phi$ is the resulting fractal code of the image $f$ by the operator $F$. An additional parameter of the operator is the list of domain image blocks $D$ involved in the code generation. There is an inverse fractal transformation that forms the image by the fractal code:

$$
f^{\prime}=F^{*}(\Phi, D)=F^{*}(F(j, D)) .
$$

To properly restore an image from its fractal code, it is important that the transformations use the same list of domain blocks $D$. In the original version, the generated fractal code is full, that means that each vertex of the graph has one parent and four descendants, but the length of the descending chain does not exceed the depth of the quad, which specified at the initial stage of the code construction. If the full tree used as the parameter for restoring the image by the operator 12 , then the resulting image $f^{\prime}$ is similar to the original image.

For obtaining modified images, incomplete trees of range blocks used. To form an incomplete tree, use the operator:

$$
\Phi^{*}=\Gamma(\Phi) .
$$


The form of the operator $\Gamma$ depends on the required result. For example, by removing some branches of a fractal tree, you can obtain an image in which the areas for which remote branches respond are smooth, and the remaining areas are clear. Using 10, the threshold operator $\Gamma$ can be represented as:

$$
\Gamma(\Phi)=\left\{R^{\prime}, D\right\}, R^{\prime}=\left\{\left.R_{i}\right|_{R}\left(D_{j}\right)>\epsilon\right\} .
$$

The operator 16 allows us to use only range blocks whose value of the membership function is greater than a given threshold $\epsilon$.
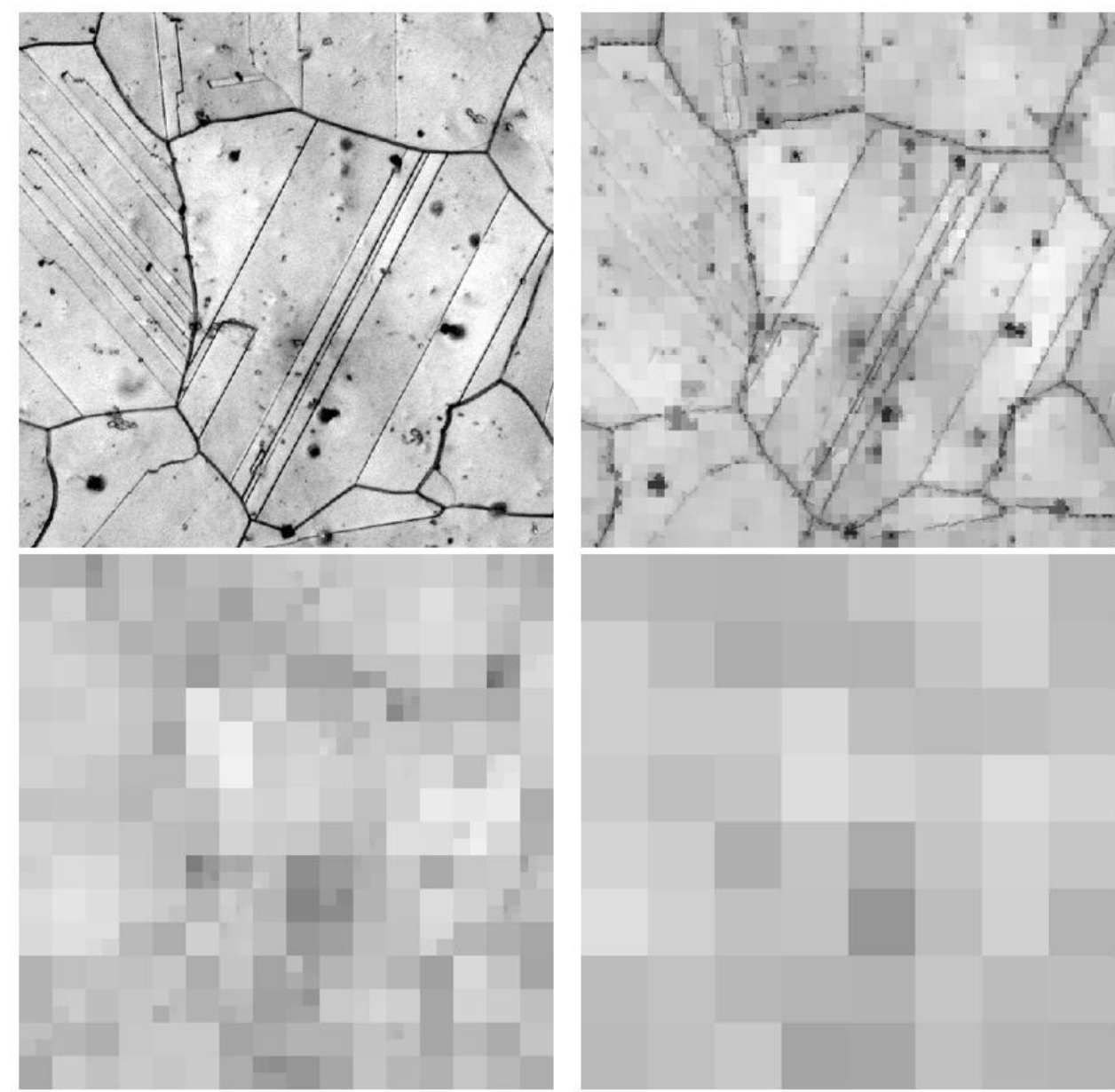

Figure 2. Fractal fuzzy representation of a test image.

To obtain a fractal representation of the image from the fractal code, need to remove the leaves of the tree. The result is a smoothed, fractal image.

Fig. 2 shows an example of such a decomposition of a test image. The resulting set of images allows for a more detailed analysis of the original image. The transition to other scales allows you to move away from small and random details. It is better to reveal the "internal" structure. It is of interest to consider the structural elements allocated at different scales of the representation of the image.

The reason for this approach is the following. Structural elements of the image, such as contours, segments, skeleton, primarily carry all the meaningful information about the original image. At the same time, they have a much more compact representation and are better suited 
for analysis, both automatic and subjective, than the original halftone image. In the case of automatic analysis, this is determined by the possibility of a formal description of the structural elements, for example, the parameters of the curve (for the contour, the skeleton) or the area geometric object (for segmentation).

\section{Detection of uncharacteristic areas in the image}

Most algorithms for digital image processing using fuzzy systems are carried out in three stages: the formation of an image fuzzification, direct processing, and, if necessary, image recovery from an image defuzzification. At the same time, the quality of image processing by such algorithms depends more on the correct choice of the fuzzy representation used [3]. The currently used algorithms for fuzzy representation of images are highly fragmented, i.e. There is no single mathematical device for constructing fuzzy models of digital images that would allow using the mathematical theory of fuzzy sets and fuzzy logic in conjunction with digital image processing methods [4].

An uncharacteristic area in the image is a section that should not be present in a given image, for example, a fracture in the image of the microstructure of the metal, a foreign object on the surface on the aerial photograph, and the like. The task of finding such areas in images is actual in metallography for the search for defects on the surfaces of materials, in radar to search for foreign objects, in medicine for the detection of foreign bodies in the blood and other areas in which image processing is actively applied [5-7].

To detect uncharacteristic blocks in the image, we formed a fractal code using a list of specific blocks instead of domain blocks.

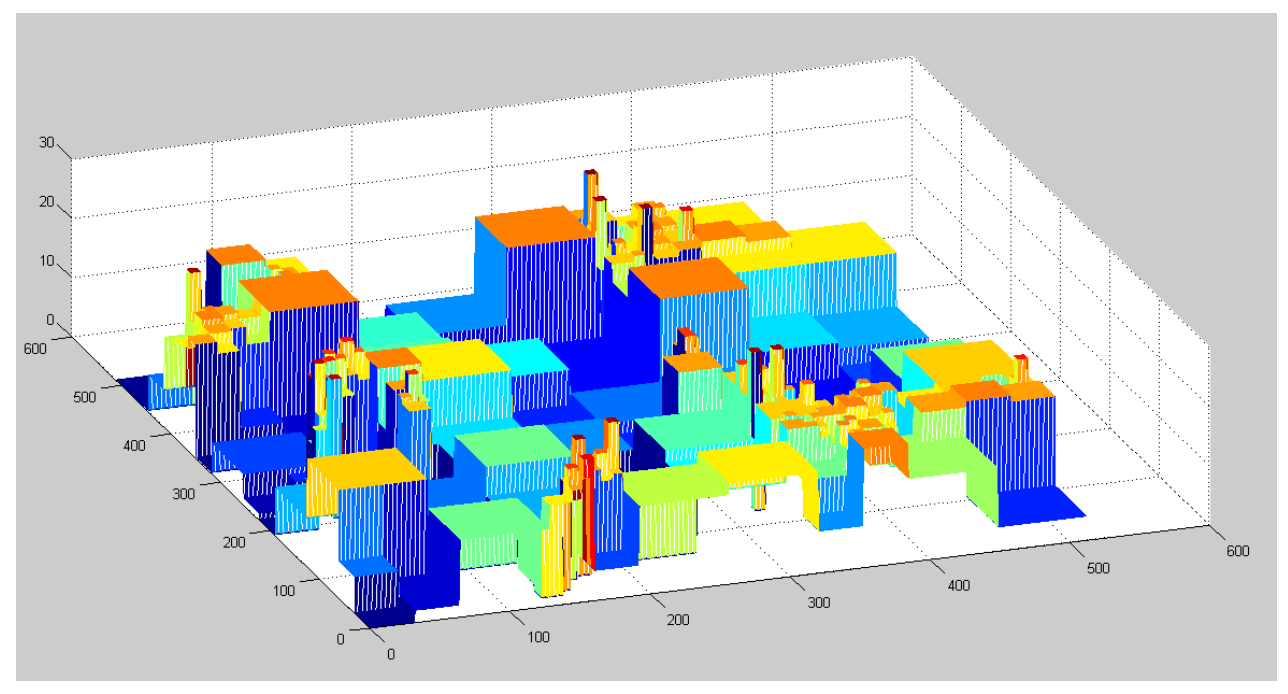

Figure 3. Three-dimensional diagram of errors in the approximation of the test image.

Fractal coding due to the use of approximation implies loss of information, since there is an error in the approximation of rank blocks by domain blocks:

$$
R_{i}=w_{i}\left(D_{j}\right)+\epsilon_{i} .
$$

In other problems this error can be neglected, because the fractal code of the image is used, without the need for decoding. In this problem, we need to obtain a vector $\{\epsilon\}=$ $\left\{\epsilon_{1}, \epsilon_{2}, \ldots, \epsilon_{k}\right\}, k=1 . . N_{R}$, then we can obtain a two-dimensional function $\Theta(x, y)$ :

$$
\Theta(x, y)=\mu\left\{\epsilon_{i} \mid(x, y) \in R_{i}\right\}
$$


where $\mu\left\{\epsilon_{i} \mid(x, y) \in R_{i}\right\}$ - the membership function that determines the amount of fuzzy uncharacteristic of the ranking block $R_{i}$ in the image.

The result is a three-dimensional diagram showing how well each block of the image approximated by the characteristic areas (Fig. 3). It is possible to establish the presence and location of image sections poorly described using specific areas, by carrying out the threshold processing of the chart

\section{Conclusion}

The fractal image code represented in the form of a graph $G$, in which the vertices of $V$ are range blocks $R$, and the arcs are the nestedness relations of range blocks. At the same time, the structure of the code remains unchanged. This approach changes only the form of representation and the mathematical apparatus of research.

The described approach to the fractal representation of images can be used to form a set of features reflecting the internal structure of the image since small details discarded. Further on, based on the proposed description, algorithms for isolating contours, image segmentation, skeletonisation can be constructed. This support the usability of the proposed algorithm in practical problems of nondestructive testing.

Also, new fractal attributes of digital images offered - specific sites. These signs show which parts of the image are involved in its formation, i.e. according to the self-similarity distribution within the image, it is possible to identify areas using which you can reproduce the image with the highest accuracy. We developed the algorithm for searching for areas of the image that are not characteristic of this class of images. The algorithm based on the fact that uncharacteristic areas of the image poorly approximated by the specific sections of this class of images.

\section{References}

[1] Mario I and Chacn M 2006 Fuzzy Logic for Image Processing: Definition and Applications of a Fuzzy Image Processing Scheme Advanced Fuzzy Logic Technologies in Industrial Applications

[2] Tamalika C 2008 Fuzzy Sets and Their Extensions: Representation, Aggregation and Models Fuzzy Measures in Image Processing

[3] Hi Z, Yan H and Pham T 1998 Fuzzy algorithms: With Applications to Image Processing and Pattern Recognition (Singapore; New Jersey; London; Hong Kong: Word Scientific) p 225

[4] Bing-Yuan C 2010 Optimal Models and Methods with Fuzzy Quantities Studies in Fuzziness and Soft Computing Fuzzy Cluster Analysis and Fuzzy Recognition 248 117-137

[5] Zhiznyakov A L, Privezentsev D G and Zakharov A A 2015 Using fractal features of digital images for the detection of surface defects Pattern Recognition and Image Analysis (Advances in Mathematical Theory and Applications) 25(1) 122-131

[6] Privezentsev D G and Zhiznyakov A L 2015 Use of specific image segments in tasks of digital image processing International Conference Stability and Control Processes in Memory of V.I. Zubov 659-660

[7] Zhiznyakov A L, Privezentsev D G and Pugin E V 2014 Use of fractal signs of digital images for detection of surface defects 24th International Crimean Conference Microwave and Telecommunication Technology Conference Proceedings 391-392

\section{Acknowledgments}

The reported study was funded by RFBR according to the research project 17-47-330073. 